Internat. J. Math. \& Math. Sci.

Vol. 23, No. 4 (2000) 253-259

S0161171200001502

(C) Hindawi Publishing Corp.

\title{
WEAK AND STRONG FORMS OF IRRESOLUTE MAPS
}

\author{
MIGUEL CALDAS CUEVA
}

(Received 23 March 1998 and in revised form 29 July 1998)

\begin{abstract}
We consider new weak and stronger forms of irresolute and semi-closure via the concept sg-closed sets which we call ap-irresolute maps, ap-semi-closed maps and contra-irresolute and use it to obtain a characterization of semi- $T_{1 / 2}$ spaces.
\end{abstract}

Keywords and phrases. Topological spaces, sg-closed sets, semi-open sets, semi-closed maps, irresolute maps.

2000 Mathematics Subject Classification. Primary 54C10, 54D10.

1. Introduction. The concept of a semi-generalized closed set (written in short as sg-closed set) of a topological space was introduced by Bhattacharyya and Lahiri [2]. These sets were also considered by various authors (e.g., Sundaram, Maki and Balachandran [15], Caldas [4] and Dontchev and Maki [9]).

In this paper, we introduce the concept of irresoluteness called ap-irresolute maps and ap-semi-closed maps by using sg-closed sets and study some of their basic properties. This definition enables us to obtain conditions under which maps and inverse maps preserve sg-closed sets. Also, in this paper, we present a new generalization of irresoluteness called contra-irresolute. We define this last class of map by the requirement that the inverse image of each semi-open set in the codomain is semi-closed in the domain. This notion is a stronger form of ap-irresoluteness. Finally, we also characterize the class of semi- $T_{1 / 2}$ spaces in terms of ap-irresolute and ap-semi-closed maps.

Throughout this paper, $(X, \tau),(Y, \sigma)$, and $(Z, \gamma)$ represent nonempty topological spaces on which no separation axioms are assumed, unless otherwise mentioned. For a subset $A$ of a space $(X, \tau), \mathrm{Cl}(A)$, and $\operatorname{Int}(A)$ denote the closure of $A$ and the interior of $A$, respectively.

2. Preliminaries. Since we require the following known definitions, notations, and some properties, we recall them in this section.

DEFINITION 2.1. A subset $A$ of a space $(X, \tau)$ is said to be semi-open [11] if there exists $O \in \tau$ such that $O \subseteq A \subseteq \mathrm{Cl}(O)$. The semi-interior [6] of $A$ denoted by $\operatorname{sint}(A)$, is defined by the union of all semi-open sets of $(X, \tau)$ contained in $A$.

REMARK 2.2. (i) A subset $A$ is semi-open [6] if and only if $\operatorname{sint}(A)=A$.

(ii) $\operatorname{sint}(A)=A \cap \mathrm{Cl}(\operatorname{Int}(A))[10]$.

By $\mathrm{SO}(X, \tau)$ we mean the collection of all semi-open sets in $(X, \tau)$.

Definition 2.3. A subset $B$ of $(X, \tau)$ is said to be semi-closed [3] if its complement $B^{c}$ is semi-open in $(X, \tau)$. The semi-closure [3] of a set $B$ of $(X, \tau)$ denoted by 
$\mathrm{sCl}_{X}(B)$, briefly $\mathrm{sCl}(B)$, is defined to be the intersection of all semi-closed sets of $(X, \tau)$ containing $B$.

REMARK 2.4. (i) A subset $B$ is semi-closed [13] if and only if $\operatorname{sCl}(B)=B$.

(ii) $\mathrm{sCl}(B)=B \cup \operatorname{Int}(\mathrm{Cl}(B))[10]$.

DEFINITION 2.5. A map $f:(X, \tau) \rightarrow(Y, \sigma)$ is called irresolute [7] if $f^{-1}(O)$ is semiopen in $(X, \tau)$ for every $O \in \mathrm{SO}(Y, \sigma)$.

Definition 2.6. A map $f:(X, \tau) \rightarrow(Y, \sigma)$ is called pre-semi-closed (resp., presemi-open) [7] if for every semi-closed (resp., semi-open) set $B$ of $(X, \tau), f(B)$ is semiclosed (resp., semi-open) in $(Y, \sigma)$.

DEFINITION 2.7. A subset $F$ of $(X, \tau)$ is said to be semi-generalized closed (written in short as sg-closed) in $(X, \tau)$ [2] if $\operatorname{sCl}(F) \subseteq O$ whenever $F \subseteq O$ and $O$ is semi-open in $(X, \tau)$. A subset $B$ is said to be semi-generalized open (written as sg-open) in $(X, \tau)$ [2] if its complement $B^{c}=X-B$ is sg-closed in $(X, \tau)$.

3. Ap-irresolute, ap-semi-closed and contra-irresolute maps. Let $f:(X, \tau) \rightarrow$ $(Y, \sigma)$ be a map from a topological space $(X, \tau)$ into a topological space $(Y, \sigma)$.

DEFINITION 3.1. A map $f:(X, \tau) \rightarrow(Y, \sigma)$ is said to be approximately irresolute (or ap-irresolute) if $\operatorname{sCl}(F) \subseteq f^{-1}(O)$ whenever $O$ is a semi-open subset of $(Y, \sigma), F$ is a sg-closed subset of $(X, \tau)$, and $F \subseteq f^{-1}(O)$.

DEFINITION 3.2. A map $f:(X, \tau) \rightarrow(Y, \sigma)$ is said to be approximately semi-closed (or ap-semi-closed) if $f(B) \subseteq \operatorname{sint}(A)$ whenever $A$ is a sg-open subset of $(Y, \sigma), B$ is a semi-closed subset of $(X, \tau)$, and $f(B) \subseteq A$.

Clearly irresolute maps are ap-irresolute and pre-semi-closed maps are ap-semiclosed, but not conversely.

The proof follows from Definition 3.1 and [2, Def. 1] (resp., Definition 3.2 and [2, Thm. 6]).

The following example shows the converse implications do not hold.

EXAMPLE 3.3. Let $X=\{a, b\}$ be the Sierpinski space with the topology, $\tau=\{\emptyset,\{a\}$, $X$ \}. Let $f: X \rightarrow X$ be defined by $f(a)=b$ and $f(b)=a$. Since the image of every semi-closed set is semi-open, then $f$ is ap-semi-closed (similarly, since the inverse image of every semi-open set is semi-closed, then $f$ is ap-irresolute). However $\{b\}$ is semi-closed in $(X, \tau)$ (resp., $\{a\}$ is semi-open) but $f(\{b\})$ is not semi-closed (resp., $f^{-1}(\{a\})$ is not semi-open in $\left.(X, \tau)\right)$. Therefore $f$ is not pre-semi-closed (resp., $f$ is not irresolute).

THEOREM 3.4. (i) $f:(X, \tau) \rightarrow(Y, \sigma)$ is ap-irresolute if $f^{-1}(O)$ is semi-closed in $(X, \tau)$ for every $O \in \mathrm{SO}(Y, \sigma)$.

(ii) $f:(X, \tau) \rightarrow(Y, \sigma)$ is ap-semi-closed if $f(B) \in \mathrm{SO}(Y, \sigma)$ for every semi-closed subset $B$ of $(X, \tau)$.

Proof. (i) Let $F \subseteq f^{-1}(O)$, where $O \in \mathrm{SO}(Y, \sigma)$ and $F$ is a sg-closed subset of $(X, \tau)$. Therefore $\mathrm{sCl}(F) \subseteq \operatorname{sCl}\left(f^{-1}(O)\right)=f^{-1}(O)$. Thus $f$ is ap-irresolute. 
(ii) Let $f(B) \subseteq A$, where $B$ is a semi-closed subset of $(X, \tau)$ and $A$ is a sg-open subset of $(Y, \sigma)$. Therefore $\operatorname{sint}(f(B)) \subseteq \operatorname{sint}(A)$. Then $f(B) \subseteq \operatorname{sint}(A)$. Thus $f$ is apsemi-closed.

This theorem was used in Example 3.3.

REMARK 3.5. Let $(X, \tau)$ denote the topological space defined in Example 3.3. Then the identity map on $(X, \tau)$ is both ap-irresolute and ap-semi-closed, it is clear that the converses of Theorem 3.4 do not hold.

In the following theorem, we get under certain conditions that the converse of Theorem 3.4 is true.

THEOREM 3.6. Let $f:(X, \tau) \rightarrow(Y, \sigma)$ be a map from a topological space $(X, \tau)$ in a topological space $(Y, \sigma)$.

(i) If the semi-open and semi-closed sets of $(X, \tau)$ coincide, then $f$ is ap-irresolute if and only if $f^{-1}(O)$ is semi-closed in $(X, \tau)$ for every $O \in \mathrm{SO}(Y, \sigma)$.

(ii) If the semi-open and semi-closed sets of $(Y, \sigma)$ coincide, then $f$ is ap-semi-closed if and only if $f(B) \in \mathrm{SO}(Y, \sigma)$ for every semi-closed subset $B$ of $(X, \tau)$.

Proof. (i) Assume $f$ is ap-irresolute. Let $A$ be an arbitrary subset of $(X, \tau)$ such that $A \subseteq Q$, where $Q \in \mathrm{SO}(X, \tau)$. Then by hypothesis $\operatorname{sCl}(A) \subseteq \operatorname{sCl}(Q)=Q$. Therefore all subsets of $(X, \tau)$ are sg-closed (and hence all are sg-open). So, for any $O \in \operatorname{SO}(Y, \sigma)$, $f^{-1}(O)$ is sg-closed in $(X, \tau)$. Since $f$ is ap-irresolute $\operatorname{sCl}\left(f^{-1}(O)\right) \subseteq f^{-1}(O)$. Therefore $\operatorname{sCl}\left(f^{-1}(O)\right)=f^{-1}(O)$, i.e., $f^{-1}(O)$ is semi-closed in $(X, \tau)$.

The converse is clear by Theorem 3.4.

(ii) Assume $f$ is ap-semi-closed. Reasoning as in (i), we obtain that all subsets of $(Y, \sigma)$ are sg-open. Therefore for any semi-closed subset of $B$ of $(X, \tau), f(B)$ is sgopen in $Y$. Since $f$ is ap-semi-closed $f(B) \subseteq \operatorname{sint}(f(B))$. Therefore $f(B)=\operatorname{sint}(f(B))$, i.e, $f(B)$ is semi-open. The converse is clear by Theorem 3.4.

As immediate consequence of Theorem 3.6, we have the following.

COROLlary 3.7. Let $f:(X, \tau) \rightarrow(Y, \sigma)$ be a map from a topological space $(X, \tau)$ in a topological space $(Y, \sigma)$.

(i) If the semi-open and semi-closed sets of $(X, \tau)$ coincide, then $f$ is ap-irresolute if and only if $f$ is irresolute.

(ii) If the semi-open and semi-closed sets of $(Y, \sigma)$ coincide, then $f$ is ap-semi-closed if and only if $f$ is pre-semi-closed.

A map $f:(X, \tau) \rightarrow(Y, \sigma)$ is called contra-irresolute if $f^{-1}(O)$ is semi-closed in $(X, \tau)$ for each $O \in \mathrm{SO}(Y, \sigma)$, and contra-pre-semi-closed if $f(B) \in \mathrm{SO}(Y, \sigma)$ for each semi-closed set $B$ of $(X, \tau)$.

REMARK 3.8. In fact, contra-irresoluteness and irresoluteness are independent notions. Example 3.3 shows that contra-irresoluteness does not imply irresoluteness while the reverse is shown in the following example.

EXAMPLE 3.9. An irresolute map need not be contra-irresolute. The identity map on the topological space $(X, \tau)$ where $\tau=\{\emptyset,\{a\}, X\}$ is an example of an irresolute map which is not contra-irresolute. 
In the same manner, we can prove that contra-pre-semi-closed maps and pre-semiclosed are independent notions.

The following result can be easily verified. Its proof is straightforward.

THEOREM 3.10. Let $f:(X, \tau) \rightarrow(Y, \sigma)$ be a map. Then the following conditions are equivalent:

(i) $f$ is contra-irresolute.

(ii) The inverse image of each semi-closed set in $Y$ is semi-open in $X$.

REMARK 3.11. By Theorem 3.4, we have that every contra-irresolute map is apirresolute and every contra-pre-semi-closed is ap-semi-closed, the converse implication do not hold.

A map $f:(X, \tau) \rightarrow(Y, \sigma)$ is called perfectly contra-irresolute if the inverse of every semi-open set in $Y$ is semi-clopen in $X$. Hence, every perfectly contra-irresolute map is contra-irresolute and irresolute.

Clearly, the following diagram holds and none of its implications is reversible:

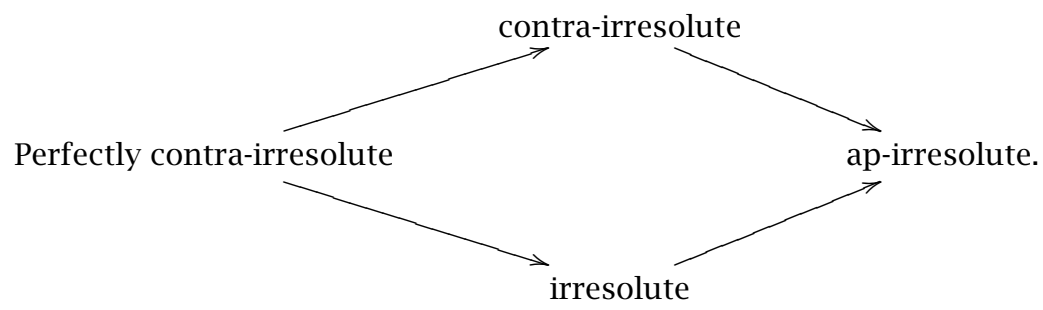

The next two theorems establish conditions under which maps and inverse maps preserve sg-closed sets.

Sundaram, Maki and Balachandran in [15, Thm. 3.7] showed that the irresolute presemi-closed inverse image of a sg-closed set is sg-closed. We strengthen this result slightly by replacing the pre-semi-closed requirement with ap-semi-closed.

THEOREM 3.12. If a map $f:(X, \tau) \rightarrow(Y, \sigma)$ is irresolute and ap-semi-closed, then $f^{-1}(A)$ is sg-closed (resp., sg-open) whenever $A$ is sg-closed (resp., sg-open) subset of $(Y, \sigma)$.

Proof. Let $A$ be a sg-closed subset of $(Y, \sigma)$. Suppose that $f^{-1}(A) \subseteq O$ where $O \in \mathrm{SO}(X, \tau)$. Taking complements we obtain $O^{c} \subseteq f^{-1}\left(A^{c}\right)$ or $f\left(O^{c}\right) \subseteq A^{c}$. Since $f$ is an ap-semi-closed and $\operatorname{sint}(A)=A \cap \mathrm{Cl}(\operatorname{Int}(A))$ and $\operatorname{sCl}(A)=A \cup \operatorname{Int}(\operatorname{Cl}(A))$, then $f\left(O^{c}\right) \subseteq \operatorname{sint}\left(A^{c}\right)=(\operatorname{sCl}(A))^{c}$. It follows that $O^{c} \subseteq\left(f^{-1}(\operatorname{sCl}(A))\right)^{c}$ and hence $f^{-1}(\mathrm{sCl}(A)) \subseteq O$. Since $f$ is irresolute $f^{-1}(\mathrm{sCl}(A))$ is semi-closed. Thus we have $\operatorname{sCl}\left(f^{-1}(A)\right) \subseteq \operatorname{sCl}\left(f^{-1}(\operatorname{sCl}(A))\right)=f^{-1}(\operatorname{sCl}(A)) \subseteq O$. This implies that $f^{-1}(A)$ is sgclosed in $(X, \tau)$. A similar argument shows that inverse images of sg-open are sg-open.

This is known (see [15]) that the semi-continuous pre-semi-closed image of a sgclosed set is sg-closed. The following theorem test this result replacing the semicontinuous requirement with ap-irresolute. 
THEOREM 3.13. If a map $f:(X, \tau) \rightarrow(Y, \sigma)$ is ap-semi-irresolute and pre-semiclosed, then for every sg-closed $F$ of $(X, \tau), f(F)$ is sg-closed set of $(Y, \sigma)$.

Proof. Let $F$ be a sg-closed subset of $(X, \tau)$. Let $f(F) \subseteq O$ where $O \in \operatorname{SO}(Y, \sigma)$. Then $F \subseteq f^{-1}(O)$ holds. Since $f$ is ap-irresolute $\operatorname{sCl}(F) \subseteq f^{-1}(O)$ and hence $f(\operatorname{sCl}(F))$ $\subseteq O$. Therefore, we have $\mathrm{sCl}(f(F)) \subseteq \operatorname{sCl}(f(\mathrm{sCl}(F)))=f(\mathrm{sCl}(F)) \subseteq O$. Hence $f(F)$ is sg-closed in $(Y, \sigma)$.

Now, reasoning as in [9], we obtain that the composition of two contra-irresolute maps need not be contra-irresolute. Really, Let $X=\{a, b\}$ be the Sierpinski space and set $\boldsymbol{\tau}=\{\emptyset,\{a\}, X\}$ and $\sigma=\{\emptyset,\{b\}, X\}$. The identity maps $f:(X, \tau) \rightarrow(X, \sigma)$ and $g$ : $(X, \sigma) \rightarrow(X, \tau)$ are both contra-irresolute but their composition $g \circ f:(X, \tau) \rightarrow(X, \tau)$ is not contra-irresolute.

However the following theorem holds. The proof is easy and hence omitted.

THEOREM 3.14. Let $f:(X, \tau) \rightarrow(Y, \sigma)$ and $g:(Y, \sigma) \rightarrow(Z, \gamma)$ be two maps such that $g \circ f:(X, \tau) \rightarrow(Z, \gamma)$. Then,

(i) $g \circ f$ is contra-irresolute, if $g$ is irresolute and $f$ is contra-irresolute.

(ii) $g \circ f$ is contra-irresolute, if $g$ is contra-irresolute and $f$ is irresolute.

In an analogous way, we have the following.

THEOREM 3.15. Let $f:(X, \tau) \rightarrow(Y, \sigma), g:(Y, \sigma) \rightarrow(Z, \gamma)$ be two maps such that $g \circ f:(X, \tau) \rightarrow(Z, \gamma)$. Then,

(i) $g \circ f$ is ap-semi-closed, if $f$ is pre-semi-closed and $g$ is ap-semi-closed.

(ii) $g \circ f$ is ap-semi-closed, if $f$ is ap-semi-closed and $g$ is pre-semi-open and $g^{-1}$ preserves sg-open sets.

(iii) $g \circ f$ is ap-irresolute, if $f$ is ap-irresolute and $g$ is irresolute.

Proof. To prove statement (i), suppose $B$ is an arbitrary semi-closed subset in $(X, \tau)$ and $A$ is a sg-open subset of $(Z, \gamma)$ for which $g \circ f(B) \subseteq A$. Then $f(B)$ is semi-closed in $(Y, \sigma)$ because $f$ is pre-semi-closed. Since $g$ is ap-semi-closed, $g(f(B))$ $\subseteq \operatorname{sInt}(A)$. This implies that $g \circ f$ is ap-semi-closed.

To prove statement (ii), suppose $B$ is an arbitrary semi-closed subset of $(X, \tau)$ and $A$ is a sg-open subset of $(Z, \gamma)$ for which $g \circ f(B) \subseteq A$. Hence $f(B) \subseteq g^{-1}(A)$. Then $f(B) \subseteq \operatorname{sint}\left(g^{-1}(A)\right)$ because $g^{-1}(A)$ is sg-open and $f$ is ap-semi-closed. Thus,

$$
(g \circ f)(B)=g(f(B)) \subseteq g\left(\operatorname{sInt}\left(g^{-1}(A)\right)\right) \subseteq \operatorname{sInt}\left(g g^{-1}(A)\right) \subseteq \operatorname{sInt}(A) .
$$

This implies that $g \circ f$ is ap-semi-closed.

To prove statement (iii), suppose $F$ is an arbitrary sg-closed subset of $(X, \tau)$ and $O \in$ $\mathrm{SO}(Z, \gamma)$ for which $F \subseteq(g \circ f)^{-1}(O)$. Then $g^{-1}(O) \in \mathrm{SO}(Y, \sigma)$ because $g$ is irresolute. Since $f$ is ap-irresolute, $\operatorname{sCl}(F) \subseteq f^{-1}\left(g^{-1}(O)\right)=(g \circ f)^{-1}(O)$. This proves that $g \circ f$ is ap-irresolute.

As a consequence of Theorem 3.15, we have the following.

COROLlary 3.16. Let $f_{\alpha}: X \rightarrow Y_{\alpha}$ be a map for each $\alpha \in \Omega$ and $f: X \rightarrow \prod Y_{\alpha}$ the product map given by $f(x)=\left(f_{\alpha}(x)\right)$. If $f$ is ap-irresolute, then $f_{\alpha}$ is ap-irresolute for each $\alpha$. 
Proof. For each $\beta$ let $P_{\beta}: \prod Y_{\alpha} \rightarrow Y_{\beta}$ be the projection map. Then $f_{\beta}=P_{\beta} \circ f$, where $P_{\beta}$ is irresolute. By Theorem 3.15(iii), $f_{\beta}$ is ap-irresolute.

Regarding the restriction $f_{A}$ of a map $f:(X, \tau) \rightarrow(Y, \sigma)$ to a subset $A$ of $X$, we have the following.

THEOREM 3.17. (i) If $f:(X, \tau) \rightarrow(Y, \sigma)$ is ap-semi-closed and $A$ is a semi-closed set of $(X, \tau)$, then its restriction $f_{A}:\left(A, \tau_{A}\right) \rightarrow(Y, \sigma)$ is ap-semi-closed.

(ii) If $f:(X, \tau) \rightarrow(Y, \sigma)$ is ap-irresolute and $A$ is an open, sg-closed subset of $(X, \tau)$, then $f_{A}:\left(A, \tau_{A}\right) \rightarrow(Y, \sigma)$ is ap-irresolute.

PROoF. (i) Suppose $B$ is an arbitrary semi-closed subset of $\left(A, \tau_{A}\right)$ and $O$ a sg-open subset of $(Y, \sigma)$ for which $f_{A}(B) \subseteq O$. By [12, Thm. 2.6] $B$ is semi-closed of $(X, \tau)$ because $A$ is semi-closed of $(X, \tau)$. Then $f_{A}(B)=f(B) \subseteq O$. Using Definition 3.2, we have $f_{A}(B) \subseteq \operatorname{sint}(O)$. Thus $f_{A}$ is an ap-semi-closed map.

(ii) Assume that $F$ is a sg-closed subset relative to $A$, i.e., sg-closed in $\left(A, \tau_{A}\right)$, and $G$ is a semi-open subset of $(Y, \sigma)$ for which $F \subseteq\left(f_{A}\right)^{-1}(G)$. Then $F \subseteq f^{-1}(G) \cap A$. By [2, Thm. 3] $F$ is sg-closed in $X$. Since $f$ is ap-irresolute $s C(F) \subseteq f^{-1}(G)$. Then $\operatorname{sCl}(F) \cap A \subseteq f^{-1}(G) \cap A$. Using the fact that $\operatorname{sCl}(F) \cap A=\operatorname{sCl}_{A}(F)$ for every pre-open subset [14, Thm. 2.4], we have $\operatorname{sCl}_{A}(F) \subseteq\left(f_{A}\right)^{-1}(G)$. Thus $f_{A}:\left(A, \tau_{A}\right) \rightarrow(Y, \sigma)$ is apirresolute.

Observe that restrictions of ap-semi-closed maps can fail to be ap-semi-closed.

Really, as in [1], let $X$ be an indiscrete space. Then $X$ and $\emptyset$ are the only semi-open subsets of $X$. Hence the semi-closed subsets of $X$ are also $X$ and $\emptyset$. Let $A$ a nonempty proper subset of $X$. The identity map $f: X \rightarrow X$ is ap-semi-closed, but $f_{A}: A \rightarrow X$ fails to be ap-semi-closed. In fact, $f(A)$ is sg-open (every subset of $X$ is sg-open) and $A$ is closed in $A$. Therefore semi-closed in $\left(A, \tau_{A}\right)$, but $f(A) \subseteq \operatorname{sInt}(f(A))$.

4. A characterization of semi- $T_{1 / 2}$ spaces. In the following theorem, we give a characterization of a class of topological space called semi- $T_{1 / 2}$ space by using the concepts of ap-irresolute maps and ap-semi-closed maps.

We recall that a topological space $(X, \tau)$ is said to be semi- $T_{1 / 2}$ space [2], if every sg-closed set is semi-closed.

THEOREM 4.1. Let $(X, \tau)$ be a topological space. Then the following statements are equivalent:

(i) $(X, \tau)$ is a semi- $T_{1 / 2}$ space.

(ii) For every space $(Y, \sigma)$ and every map $f:(X, \tau) \rightarrow(Y, \sigma), f$ is ap-irresolute.

Proof. (i) $\Longrightarrow$ (ii): Let $F$ be a sg-closed subset of $(X, \tau)$ and suppose that $F \subseteq f^{-1}(O)$, where $O \in \mathrm{SO}(Y, \sigma)$. Since $(X, \tau)$ is a semi- $T_{1 / 2}$ space, $F$ is semi-closed (i.e., $F=\operatorname{sCl}(F)$ ). Therefore $\mathrm{sCl}(F) \subseteq f^{-1}(O)$. Then $f$ is ap-irresolute.

(ii) $\Longrightarrow$ (i): Let $B$ be a sg-closed subset of $(X, \tau)$ and let $Y$ be the set $X$ with the topology $\sigma=\{\emptyset, B, Y\}$. Finally let $f:(X, \tau) \rightarrow(Y, \sigma)$ be the identity map. By assumption $f$ is ap-irresolute. Since $B$ is sg-closed in $(X, \tau)$ and semi-open in $(Y, \sigma)$ and $B \subseteq f^{-1}(B)$, it follows that $\mathrm{sCl}(B) \subseteq f^{-1}(B)=B$. Hence $B$ is semi-closed in $(X, \tau)$ and therefore is semi- $T_{1 / 2}$. 
THEOREM 4.2. Let $(Y, \sigma)$ be a topological space. Then the following statements are equivalent:

(i) $(Y, \sigma)$ is a semi- $T_{1 / 2}$ space.

(ii) For every space $(X, \tau)$ and every map $f:(X, \tau) \rightarrow(Y, \sigma), f$ is ap-semi-closed.

Proof. Analogous to Theorem 4.1 making the obvious changes.

We refer the reader to $[2,4,5,15]$ for other results on semi- $T_{1 / 2}$ spaces.

\section{REFERENCES}

[1] C. W. Baker, On preserving g-closed sets, Kyungpook Math. J. 36 (1996), no. 1, 195-199. MR 97d:54026. Zbl 908.54011.

[2] P. Bhattacharyya and B. K. Lahiri, Semi-generalized closed sets in topology, Indian J. Math. 29 (1987), no. 3, 375-382. MR 90a:54004. Zbl 687.54002.

[3] N. Biswas, On characterizations of semi-continuous functions, Atti Accad. Naz. Lincei Rend. Cl. Sci. Fis. Mat. Natur. (8) 48 (1970), 399-402. MR 44\#998. Zbl 199.25602.

[4] M. Caldas, Semi-T $1 / 2$ spaces, Pro-Math. VIII (1994), 115-121.

[5] _ A separation axiom between semi-T $T_{0}$ and semi-T $T_{1}$, Mem. Fac. Sci. Kôchi Univ. Ser. A Math. 18 (1997), 37-42. MR 98c:54015. Zbl 893.54014.

[6] S. G. Crossley and S. K. Hildebrand, Semi-closure, Texas J. Sci. 22 (1971), 99-112.

[7] Semi-topological properties, Fund. Math. 74 (1972), no. 3, 233-254. MR 46846. Zbl 206.51501.

[8] J. Dontchev, Contra-continuous functions and strongly S-closed spaces, Internat. J. Math. Math. Sci. 19 (1996), no. 2, 303-310. MR 96m:54021. Zbl 840.54015.

[9] J. Dontchev and H. Maki, On sg-closed sets and semi- $\lambda$-closed sets, Questions Answers Gen. Topology 15 (1997), no. 2, 259-266. MR 98h:54002. Zbl 990.12413.

[10] D. S. Jankovic and I. L. Reilly, On semiseparation properties, Indian J. Pure Appl. Math. 16 (1985), no. 9, 957-964. MR 87a:54029. Zbl 572.54010.

[11] N. Levine, Semi-open sets and semi-continuity in topological spaces, Amer. Math. Monthly 70 (1963), 36-41. MR 29\#4025. Zbl 113.16304.

[12] S. N. Maheshwari and R. Prasad, On s-regular spaces, Glas. Mat. Ser. III 10 (30) (1975), no. 2, 347-350. MR 53 1518. Zbl 316.54017.

[13] T. Noiri, A note on semi-continuous mappings, Atti Accad. Naz. Lincei Rend. Cl. Sci. Fis. Mat. Natur. (8) 55 (1974), 400-403. MR 51 4139. Zbl 336.54012.

[14] T. Noiri and B. Ahmad, A note on semi-open functions, Math. Sem. Notes Kobe Univ. 10 (1982), no. 2, 437-441. MR 84g:54015. Zbl 515.54010.

[15] P. Sundaram, H. Maki, and K. Balachandran, Semi-generalized continuous maps and semi$T_{1 / 2}$ spaces, Bull. Fukuoka Univ. Ed. Part III 40 (1991), 33-40. MR 92j:54032c. Zbl 790.54010 .

CAldas: Departamento of Matemática, Aplicada-IMUfF, Universidade Federal FlumiNENSE, RUA MÁRIO SANTOS BRAGA $s / n^{0}$, CEP: 24020-140, NitERÓI RJ, BRASIL

E-mail address: gmamccs@vm.uff.br 


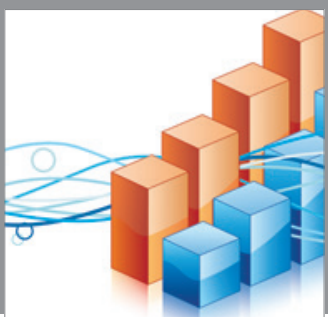

Advances in

Operations Research

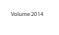

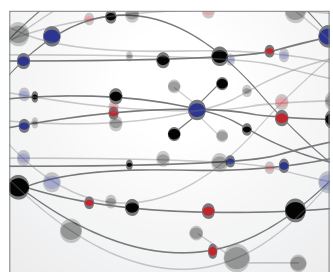

\section{The Scientific} World Journal
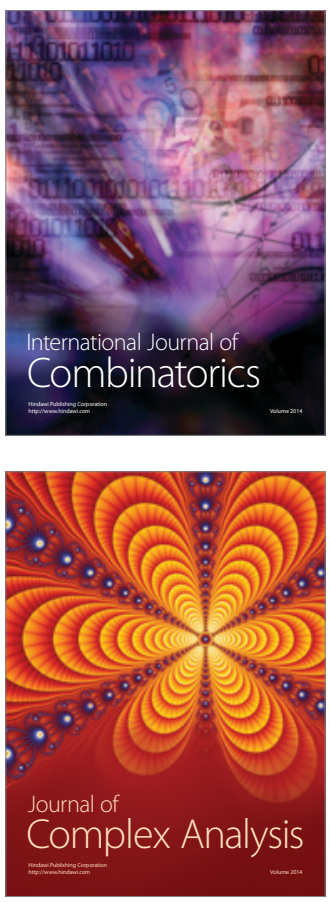

International Journal of

Mathematics and

Mathematical

Sciences
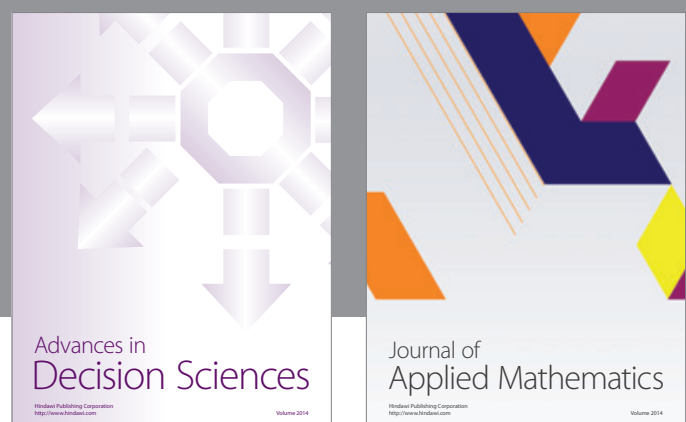

Journal of

Applied Mathematics
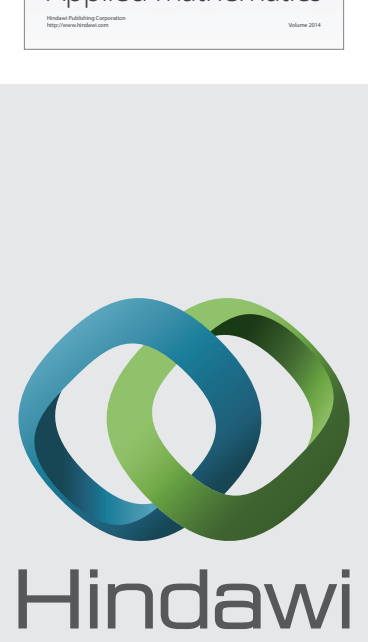

Submit your manuscripts at http://www.hindawi.com
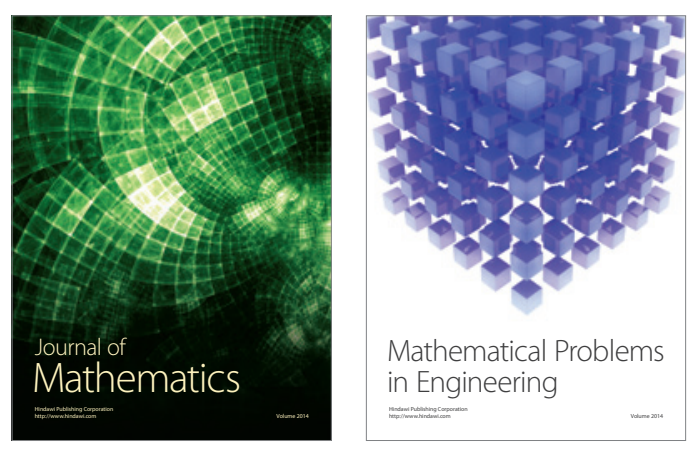

Mathematical Problems in Engineering
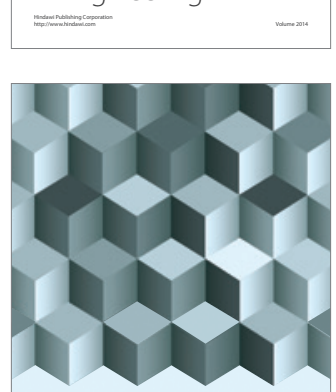

Journal of

Function Spaces
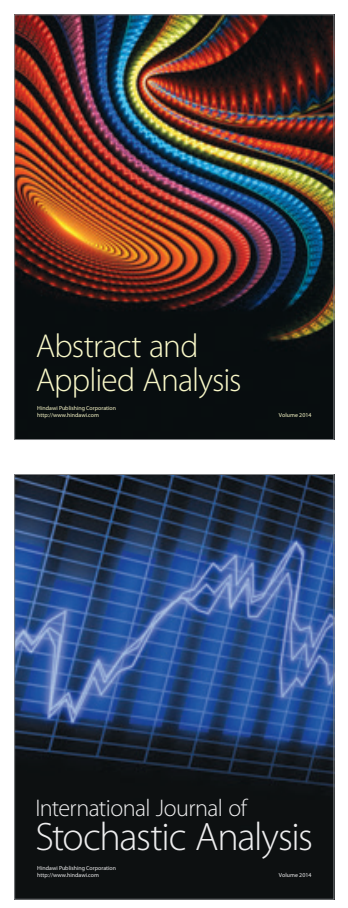

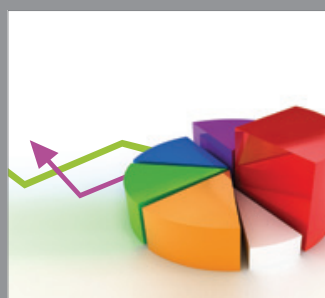

ournal of

Probability and Statistics

Promensencen
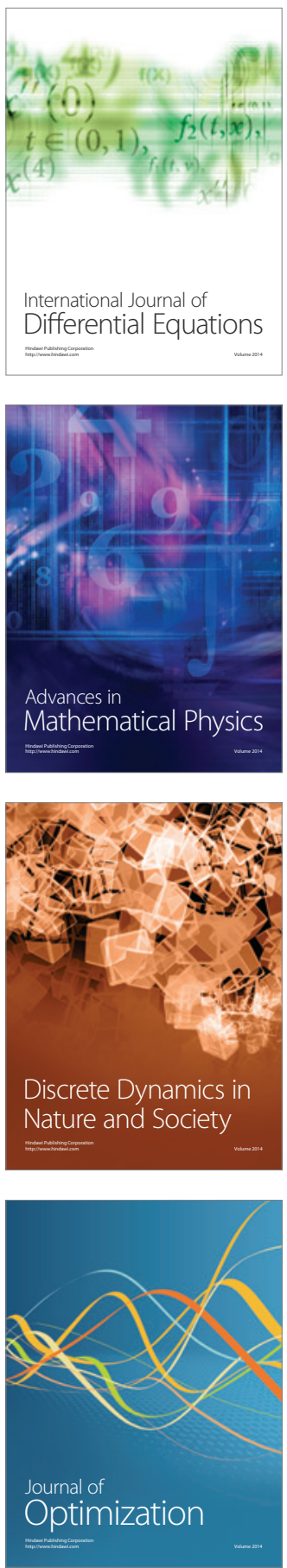